\title{
The Formidable Task of Developing a Predictive Capability of the Space Environment of the Solar System
}

\author{
L. A. Fisk \\ Department of Atmospheric, Oceanic, and Space Sciences \\ University of Michigan, Ann Arbor, Michigan 48109
}

\begin{abstract}
We have before us a rather formidable task - we are in need of developing a predictive capability of the space environment of the solar system. Such a capability is required to protect our everincreasing assets in space, and to understand the influence of the Sun on Earth. Such a capability will be essential if we are to send humans forth on long-duration space flights. This is not a problem that can be undertaken haphazardly. The Sun and the space environment of the solar system are an integrated system, which needs to be studied through coordinated missions, coordinated data analysis, and coordinated theory and modeling efforts. And there needs to be a community of dedicated researchers who understand the magnitude of the task and are committed and supported to achieve the required goals. Nothing less will succeed. The complexity of the problem of developing a predictive capability of the space environment of the solar system and the requirements for success will be discussed.
\end{abstract}

\section{Introduction}

Lets us begin by fast-forwarding 20 , or 30 , or 40 years to a time when humans are conducting routine observations in deep space. There are people on the surface of the moon or perhaps Mars, performing operations at a distance from any safe haven. There are astronauts who need to be outside of their spacecraft for some periods of time. The people are supported by complex electronic hardware that is essential for their survival, and communications need to be continuous and routine.

Suppose then that whoever is in charge of these operations in deep space turns to the community of solar and space physicists, such as is represented in this symposium, and says is it safe? Is the space environment on that day, which basically means the radiation environment from energetic particles, sufficiently benign to protect both the immediate and the long-term health of the space explorers? If we were asked that question today, we would answer it by probability and analogy. There is only a certain probability that a large solar flare will occur at the time of the solar cycle when the question is asked. Perhaps we would say that the Sun, with similar levels of activity in the past, has not produced a major event.

I question, however, whether such an answer will be acceptable. We have witnessed two times in recent history the impact on space exploration of the death of space crews, with the Challenger and the Columbia accidents. Imagine what would be the impact if crews on the surface of the moon or en route to Mars were to be irreparably damaged by unpredicted space radiation.

We should ask ourselves, then, what will it take over the intervening years before routine space operations in deep space to understand the processes by which space radiation is produced, so that we can predict with near certainty the environment in which humans will fly. It is a formidable problem, but not insurmountable, as we will discuss in this paper.

\section{Types of Space Radiation}

Space radiation from energetic particles comes in three basic types: galactic cosmic rays; impulsive solar flares and the superthermal background; and large gradual solar flares:

Galactic Cosmic Rays. The galaxy and perhaps the universe are filled with energetic particles known as galactic cosmic rays. They are believed to be accelerated primarily by supernovae explosions, and they permeate the local interstellar medium outside our solar system ${ }^{1}$. As the particles propagate upstream against the outward flowing solar

\footnotetext{
* Professor L. A. Fisk, Department of Atmospheric, Oceanic, and Space Sciences, 2455 Hayward Street, Ann Arbor, MI, 48109, AIAA Member
} 
wind, the intensity of the lower energy cosmic rays, e.g. less than about $2 \mathrm{GeV} / \mathrm{nucleon}$ ions, is reduced and controlled by the solar wind ${ }^{2}$. We say the cosmic ray intensity is modulated by the solar wind.

We know a great deal about galactic cosmic rays having measured over many decades, from the ground, balloons, and space, all aspects, including the spectrum [over 32 orders of magnitude in intensity and 18 orders of magnitude in energy], and the composition of all major elements and many isotopes. We also know the time variation of the cosmic rays during the solar cycle, which at the peak of the ion spectrum at several 100 $\mathrm{MeV} /$ nucleon, is typically a factor of 2-4 from solar minimum to solar maximum conditions.

The impact on human space operations or on electronic equipment from the radiation from galactic cosmic rays is thus not a space physics problem, but rather a biological and engineering problem. Galactic cosmic rays are always present, and do not vary widely. It is necessary then to determine how much accumulated radiation humans can tolerate, and whether this tolerance is affected by the circumstances of space such as microgravity, and what engineering solution such as shielding is required to achieve an acceptable level of tolerance.

There is one proviso to this conclusion. The activity level of the Sun varies from solar cycle to solar cycle, and in historical times there have been extended periods of extremely low solar activity and commensurately high levels of galactic cosmic rays, beyond those seen during the space age. To predict such long-term variations requires much better knowledge of what causes the changes in solar activity [about which we have very little knowledge] and what causes galactic cosmic ray modulation [about which we have considerable but still limited knowledge, because so much of the modulation occurs in the very outer solar system, beyond most of our observations].

Impulsive solar flares and the superthermal background. There are many small flares on the Sun, the most common of which are impulsive solar flares, of which about 1000 per year occur at solar maximum. The ion energies are typically less than about $10 \mathrm{MeV} /$ nucleon; the events are short-lived; and each event is confined to a narrow range of solar longitudes. The term solar flare is often used synonymously with these events although technically the flare is the photon radiation from the Sun caused by energetic particles, particularly electrons, raining down on the solar surface, and the energetic particle events about which we are concerned are the escaping particles, particularly ions. The more correct term for the escaping particles is Solar Energetic Particles, or SEP events.

Impulsive SEP events are fascinating events, particularly because of their unusual composition ${ }^{3}$. Helium-3 is a rare isotope of Helium [one part in 10,000] and yet is can be as abundant as the main isotope Helium-4 in impulsive SEP events; there can be equally strong enhancements in the partially-charged heavier elements, with large mass-tocharge ratios. These usual features provide important insights into the mechanisms that accelerate energetic particles at the Sun. However, the impulsive SEP events themselves are of sufficiently low intensity that they should not be of much impact on space operations.

The impulsive SEP events do provide a background of particles that can be accelerated in the larger solar flare events we discuss next. Also astrophysical plasmas in general, such as the plasma in the solar corona or in the solar wind, appear to always contain a superthermal population of energetic particles, usually with some form of power law spectrum. There is a background then of energetic particles always present on which other acceleration mechanisms can act.

Gradual Solar Flares. The solar events that we need to be concerned with are the so-called gradual solar flares, or more appropriately gradual SEP events. In these events, of which there may be 10-20 per year during solar maximum, the intensity of energetic ions rises abruptly and persists for days spread over wide solar longitudes. The energies of the particles, including the ions, can extend to relativistic energies [greater than $1 \mathrm{GeV} / \mathrm{nucleon}$ ], and these particles can arrive at Earth, following the solar disturbance, within minutes, at the relativistic travel time of $\sim$ 10 minutes. Gradual SEP events can provide a lethal dose of radiation to an exposed astronaut. Such an event occurred last January and produced the most energetic particles of any event ever measured.

The currently accepted mechanism for producing gradual SEP events is as follows ${ }^{4}$ : A large volume of mass, known as a Coronal Mass Ejection, is released from near the surface of the Sun and accelerates into the solar corona. It obtains supersonic [or super Alfvenic] speeds and drives a shock wave ahead. The shock wave accelerates energetic particles to high energies by a mechanism known as diffusive shock acceleration, and the particles are released to escape into the solar system across the broad front of the shock.

It needs to be noted first that although this mechanism is plausible and supported by observations, it is not proven. In fact there are some disquieting problems. In a recent event seen by the RHESSI spacecraft, the particles that escaped into space, presumably generated by the shock, had the identical spectrum to the particles raining downward on the solar surface [presumably generated within the solar flare]. A coincidence, perhaps, or is there a relationship between the flare particles and the escaping particles that we don't understand. It is also true that despite the wide acceptance of the mechanism of diffusive shock acceleration, there are very few examples of it working as predicted in cases where we can actually observe the shock, such as traveling shocks in the solar wind. 
Even if we take the scenario for generating gradual SEP events as valid, predicting their occurrence is challenging, for the following phenomena need to be understood and predicted:

- Where and when will a Coronal Mass Ejection occur?

- How large will it be and how will it evolve as it propagates through the corona?

- What are the upstream conditions through which it propagates and thus what will be the strength of the shock that it drives and the conditions that will determine its effectiveness in accelerating particles?

- What is the seed population of energetic particles present, to be accelerated?

It is important to recognize how little we know. A case in point is the upstream conditions, which depend primarily on the magnetic field in the outer corona, which cannot be observed with current techniques; nor can we observe the seed populations of energetic particles.

\section{What do we need to do?}

To develop a comprehensive and predictive understanding of the space environment we obviously require a coordinated effort of observation, theory, and modeling:

Observations. We currently make observations of the photon emission of the Sun, i.e. remote sensing observations of the solar surface and solar atmosphere, and we also observe the plasma and energetic particle environments in the heliosphere and the near-space environment of Earth. For example, Ulysses observes the allimportant three dimensional environment of the heliosphere; $A C E$ and WIND observe the conditions in the solar wind immediately upstream from Earth; STEREO is about to be launched to observe both remotely and in situ the evolution of Coronal Mass Ejections in the heliosphere; Voyager is observing the distant heliosphere and it will be supplemented by IBEX's observations of Energetic Neutral Atoms generated by the energetic particles in the distant heliosphere; a suite of near-Earth missions continues to study the Earth's magnetosphere and will be replaced with such missions as the Radiation Belt Storm Probes of the Living with a Star program. The SOHO mission has made profound observations of the Sun, both its surface processes and atmospheric properties. Shortly we will launch its replacement the Solar Dynamics Observatory and such important high-resolution missions such as Solar-B. Clearly we need to get the maximum information out of all these missions as long as they can effectively operate.

We need to recognize, however, that although the existing and upcoming data sets are extensive, they are still inadequate. In the case of gradual SEP events, for example, many of the important physical processes occur in the inner corona, where observational knowledge is scarce. The magnetic field, for example, which governs many aspects of the acceleration of energetic particles is unobserved. We can model; we can infer from other remote sensing observations. There are also new observational techniques, some involving ground-based instrumentation, that may some day be able to make direct observations of the coronal magnetic field. However, no matter what modeling or observational advances we make, we will never be sure of what we are inferring or seeing until we actually fly through the inner solar corona with the proposed Solar Probe mission. Solar Probe will provide a limited sample of the inner corona, but it is a sample, which if taken properly, will validate what we think is occurring and how to interpret remote observations, or more likely will profoundly alter our concepts of how the solar atmosphere behaves.

We also need to recognize the inadequacy of our spatial coverage. The solar atmosphere, which determines the space environment of the heliosphere, behaves as an integrated global system. Coronal Mass Ejections are large and produces disturbances and consequences throughout the solar atmosphere. The magnetic field of the Sun produces a global coupling of solar phenomena. Yet, we observe particularly the particle emissions from the Sun only at widely separated locations; we remotely observe the Sun mainly from Earth, which clearly limits the global coverage. There is an essential need then for the Sentinel missions, which are to provide solar and heliospheric observations from multiple locations spread in solar longitude and where possible are located closer to the Sun than the orbit of Earth.

The magnitude of our observational needs demand international cooperation in this endeavor. It is unlikely that the U.S. can afford all the needed observations. This scientific discipline, perhaps more than others, involves relatively simple and well-proven instrumentation, well within the capabilities of even developing space programs. The effort to understand the space environment of the solar system is a natural one for international cooperation; indeed, if we are to encourage the exploration of space not just as an American effort, but rather one pursued by our civilization, the study and the understanding of the space environment is are area to which many nations could contribute.

Finally, it is important to note that the observations from current, planned, and required future missions need always to be treated as a coordinated set, each revealing a different aspect of the same phenomena -- the solar control of our space environment. Each mission is a sensor of a complicated and integrated system. The data 
should always be analyzed and interpreted in the context of and in coordination with what other sensors are revealing. Missions are sold in NASA as stand-alone entities, for fear that their reliance on other missions implies an apparent increase in their cost. We should eliminate that mindset. We are assembling a comprehensive observing system, where each new mission is justified by how it strengthens what has already been assembled.

Theory. It is important to recognize that we do not understand the physical processes by which the Sun controls the space environment; we do not understand the physical processes that are responsible for gradual SEP events. We have theories, most of which are primitive. We have accepted concepts, but unfortunately if we probe the basis that led to their acceptance, it is longevity. The ideas have been around a long time, and have useful features, but if you ask whether they were ever subjected to rigorous scientific validation, rarely if ever is that so.

The space environment problem is often portrayed as analogous to the problem of weather on Earth; we even use the term Space Weather. However, in the case of weather on Earth, we know the governing physical processes of the Earth's atmosphere; we have an agreed upon set of equations that has been validated by direct observation. The Earth's weather problem is then one of developing ever more comprehensive models, and methods to assimilate data into the models. In the case of the space environment, we don't have an agreed upon set of governing equations.

There is a need then for a comprehensive theoretical analysis of where we are with understanding the physical processes that govern the space environment. Each concept should be reexamined and validated if possible, left for future testing if currently untestable; discarded if unsupported; replaced if a better concept can be developed.

Modeling. Over the last few years large scale numerical models have been developed to simulate the space environment. This is an important effort because such numerical models are needed to describe the complicated three-dimensional and dynamic phenomena in space. Such models are basically the codification of our understanding, and will be used for the predictions that we will require.

The models today, however, have several important limitations. A model is only as good as its boundary conditions, and we are currently limited in our knowledge of the relevant boundary conditions by inadequate observations. The models are also at best a crude approximation to the governing physics. The models are usually based on some form of MHD, which makes simplifying assumptions about the particle distribution functions; it does not include more complicated kinetic properties; it does not include certain dissipative processes; it is hard pressed to adequately describe the behavior of energetic particles, which rarely behave according to the simplifying assumptions. The current models then require advances in theory, which are needed to provide a better description of the underlying microphysics, and to provide useful ways to incorporate the microphysics back into the numerical models.

It is clear then that a three-prong attack on the space environment problem is required: (1) more comprehensive observations; (2) a comprehensive examination of the underlying theories, both to validate and replace current concepts and to produce useful descriptions of the microphysical processes needed for the numerical models; and (3) the development of comprehensive models which incorporate the new theoretical concepts and processes, and which rely on and are validated against the observations.

\section{A New Mindset}

Space science is usually not conducted with urgency. There is an urgency to develop hardware to meet launch windows; there can be an urgency to be the first to reveal some new phenomena. Rarely, however, is there an urgency to solve a particular problem. Indeed, a cynic would argue that solving a problem has the consequence of making enjoyable funding unnecessary.

If we are really serious about having routine operations in deep space with humans, we need to make a dramatic improvement in our ability to predict the space environment through which we will fly, and we no longer have the luxury of a leisurely research effort. There is an urgency to succeed. We require then that the national space programs recognize the importance of this problem and apply the necessary resources. And, perhaps most important, we require dedicated researchers, who believe that the problem is solvable and are willing to devote their talent and energy to its expeditious solution. When John Kennedy gave his famous speech to Congress challenging the nation to go the moon within ten years, there was an often overlooked additional paragraph in which Kennedy stated that "every scientist, every engineer, every technician, contractor, and civil servant must give his personal pledge that this nation will move forward, with the full speed of freedom, in the exciting adventure of space". I would argue to my own solar and space physics community that we should take a similar pledge and deliver a predictive capability of the space environment of the solar system. 


\section{References}

1 e.g, Diehl, R., Parizot, R., Kallenbach, R., and von Steiger, R., "The Astrophysics of Galactic Cosmic Rays", Space Science Series of ISSI, Vol. 13, Kluwer Academic Publishers, 2001.

2 e.g., Fisk, L. A., Jokipii, J. R., Simnett, G., von Steiger, R., and Wentzel, K.-P., "Cosmic Rays in the Heliosphere”, Space Science Series of ISSI, Vol. 3, Kluwer Academic Publishers. 1998.

3 e.g., Reames, D. V., "What we don't understand about ion acceleration in solar flares", in High Energy Solar Physics: Anticipating HESSI. Ed. by R. Ramaty and N. Mandzhavidze, ASP Conference Series, Vol., 206, p12, 2000

4 e.g., Reames, D., "Solar energetic particles: A paradigm shift”, Reviews of Geophysics Supplement, $33,1995$. 